\title{
End-stage Renal Disease Due to Membranous Lupus Nephritis: Is Antiphospholipid Syndrome Misdiagnosed or Underestimated?
}

\section{Mabel Aoun ${ }^{\star}$, Georges Khalil and Georges Aftimos}

Department of Nephrology, Saint-Georges Hospital Ajaltoun, Lebanon

"Corresponding author: Mabel Aoun, Department of Nephrology, Saint-Georges Hospital Ajaltoun, Lebanon, Tel: 9613351588; E-mail: aounmabel@yahoo.fr

Rec date: May 25, 2014, Acc date: June 24, 2014, Pub date: June 30, 2014

Copyright: (c) 2014 Aoun M, et al. This is an open-access article distributed under the terms of the Creative Commons Attribution License, which permits unrestricted use, distribution, and reproduction in any medium, provided the original author and source are credited.

\begin{abstract}
We report a case of a 45-year-old woman who presented for nephrotic syndrome and anemia. Her kidney biopsy confirmed the diagnosis of class $V$ membranous lupus nephritis. She had also criterias for antiphospholipid syndrome: miscarriages and antiphospholipid antibodies. She received as immunosuppression treatment corticosteroids and mycophenolate mofetil. Her kidney function deteriorated and three bolus of cyclophosphamide were given. She developed a severe pneumonia and became uremic. She underwent two sessions of acute hemodialysis and developed deep venous thrombosis in her leg. Anticoagulation was then started and her kidney function stabilized for a few months until she was put on chronic dialysis three years and a half after her initial presentation. We emphasize on the factors that worsen the renal prognosis in membranous lupus nephritis patients. We also highlight the importance of recognizing early the antiphospholipid syndrome in lupus nephritis in order to treat it and prevent eventually end-stage renal failure.
\end{abstract}

Keywords: Membranous lupus nephritis; Antiphospholipid syndrome; Renal prognosis

\section{Introduction}

Lupus nephritis (LN) is staged according to the classification revised by the International Society of Nephrology (ISN) and the Renal Pathology Society (RPS) in 2003: Class I - Minimal mesangial lupus nephritis; Class II - Mesangial proliferative lupus nephritis; Class III - Focal lupus nephritis (active and chronic; proliferative and sclerosing); Class IV - Diffuse lupus nephritis (active and chronic; proliferative and sclerosing; segmental and global); Class $\mathrm{V}-$ Membranous lupus nephritis (MLN); Class VI - Advanced sclerosis lupus nephritis [1].

Class V is found in $10-20 \%$ of patients with LN. Although regarded as indolent compared to class III and IV, class V LN is still associated with the development of ESRD. The overall probability of renal survival in patients with all classes of LN was found to be $80 \%$ at 10 years in the italian lupus nephritis study group. But the presence of diffuse proliferative nephritis in this cohort was associated with a worse prognosis [2]. Studies of pure membranous lupus nephritis patients suggested a better renal survival rate around $90 \%$ at 10 years after diagnosis $[3,4]$. Many independent risk factors for developing ESRD in class V LN were identified. Severe tubular-interstitial lesions on kidney biopsy, nephrotic range proteinuria and refractoriness to treatments predicted worse renal outcomes in a Chinese cohort of 100 patients [3]. A french study of 66 patients found that the only predictor of ESRD was the haemoglobin level [4].

Another factor that has been described to contribute to the poor prognosis of lupus nephritis is the antiphospholipid syndrome (APS) $[5,6]$. APS in patients with systemic lupus erythematosis (SLE) can occur in addition to or independent of LN.
This raises two questions: first, whether APS is always diagnosed in LN patients and second, if early recognition of APS would improve the renal outcome of those patients.

We report here a case of membranous lupus nephritis where the poor renal outcome was most propbably due to the concomitant presence of APS.

\section{Case Report}

A 45-year-old caucasian woman presented to the hospital due to fatigue and pallor. Her past medical history include an episode of "cutaneous lupus" about 10 years ago treated with a quinine for one year. She had three children and experienced three spontaneous abortions. She had no diabetes, no hypertension. No history of kidney disease, bleeding, joint swelling or deep venous thrombosis was identified. She was allergic to aspirin. On admission her physical examination revealed pale skin and conjunctives and normal blood pressure of 110/80 mmHg. No ankle edema, nor cutaneous lesions were found.

Her laboratory tests showed a hemoglobin level of 7,9 g/dL, a white blood cell count $3.9^{*} 10^{9} / 1$, platelets $329^{*} 10^{9} / 1$ and serum creatinine 1.5 $\mathrm{mg} / \mathrm{dL}$. Serum calcium and phosphate levels were normal. Serum albumin level was $35 \mathrm{~g} / \mathrm{l}$. She had high LDL Cholesterol, high triglycerides. Her urinalysis revealed proteinuria $3+$ and five red blood cells per field with no casts. Urine protein/creatinine ratio was 4700 $\mathrm{mg} / \mathrm{g}$. Antinuclear antibodies were elevated 1:360 and anti-doublestranded DNA levels were not detectable. Her serum complement levels revealed normal C3 of $99 \mathrm{mg} / \mathrm{dL}$ (Normal=50-90) and low C4 of $5 \mathrm{mg} / \mathrm{dl}$ (Normal=10-40). Absolute reticulocyte count was 75000/ $\mathrm{mm} 3$, she had high levels of serum lactate dehydrogenase (LDH) and non-detectable haptoglobin levels. She had a positive direct Coombs test and high antiphopholipid (aPL) antibodies. Cryoglobulines were negative. Serologic tests for HIV, hepatitis B and C were all negative. Her Kidney ultrasound was normal. 
Citation: Aoun M, Khalil G, Aftimos G (2014) End-stage Renal Disease Due to Membranous Lupus Nephritis: Is Antiphospholipid Syndrome Misdiagnosed or Underestimated?. J Nephrol Ther 4: 172. doi:10.4172/2161-0959.1000172

Page 2 of 3

A kidney biopsy was performed and 6 glomeruli were examined on light microscopy. One glomerulus was completely sclerotic and the remaining five showed a slight increase in mesangial matrix on trichrome staining and diffuse basement membrane thickening with on silver staining (Figures 1 and 2). Subepithelial deposits were noted on Masson trichrome staining (Figure 1). Moderate tubular atrophy, moderate interstitial infiltrates and mild fibrosis were identified on the trichrome and the periodic acid Schiff stained sections also (Figures 1 and 3). Regarding the vessels, hyaline deposits were identified in arterioles and lesions of arteriosclerosis were found in an interlobular artery. The immunofluorescence examination showed diffuse granular staining for IgG (1+), IgM (1+) and few C3. No staining for C1q nor fibrin was found. No granular staining of any immunoreactant was evident along tubular basement membranes nor in vessels.

She was put on corticosteroids $1 \mathrm{mg} / \mathrm{kg} / \mathrm{d}$ and mycophenolate mofetil $3 \mathrm{~g} / \mathrm{d}$. Angiotensin II antagonist was introduced three months later because of persistence of proteinuria. At the sixth month of her treatment she presented with persistent proteinuria $(3 \mathrm{~g} / \mathrm{d})$ and a creatinine of $1.3 \mathrm{mg} / \mathrm{dl}$, normal C3 and C4 and negative ANA. She had frequent hospitalisations for dehydration, recurrent sinusitis and recurrent cystitis treated with antibiotics. After 18 months of her diagnosis her creatinine increased to $2 \mathrm{mg} / \mathrm{dl}$ and proteinuria remained between 2 and $3 \mathrm{~g} / \mathrm{d}$. A second kidney biopsy was performed and was identical to the first one except for worsening of vascular arteriosclerosis and interstitial fibrosis (Figure 4). Mycophenolate mofetil was stopped and she received three bolus of intravenous cyclophosphamide on a monthly basis. She developed just after the third dose of cyclophosphamide a severe pneumonia and deteriorated further more her renal function. She received then broad spectrum antibiotics and a $500 \mathrm{mg}$ bolus of methylprednisolone in order to rescue her kidney function. But she became extremely uremic with a BUN serum level of $200 \mathrm{mg} / \mathrm{dl}$ and underwent two acute sessions of haemodialysis through a femoral catheter. Three days later she developed deep venous thrombosis of her leg and she was put on anticoagulant. Creatinine stabilized and dialysis was stopped. Hydroxychloroquine was added $200 \mathrm{mg} / \mathrm{d}$. Creatinine remained stable around $2.5 \mathrm{mg} / \mathrm{dl}$ for three months then she deteriorated over a year. Chronic hemodialysis was initiated after three years and a half of her initial presentation.

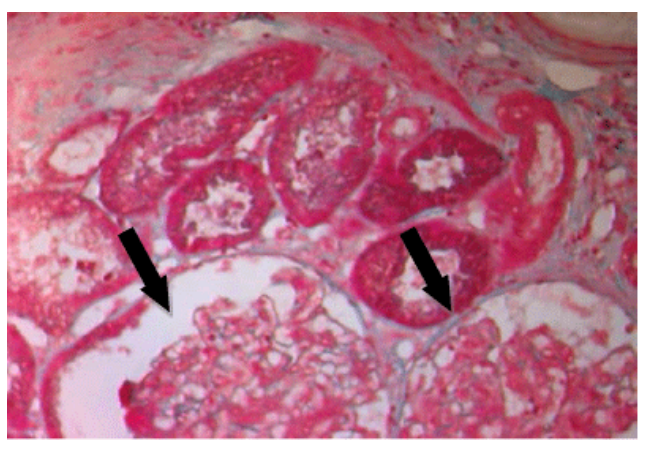

Figure 1: First kidney biopsy. Two glomeruli with mesangial expansion and subepithelial hyaline deposits ( Masson trichrome stain, $\times 200$ )

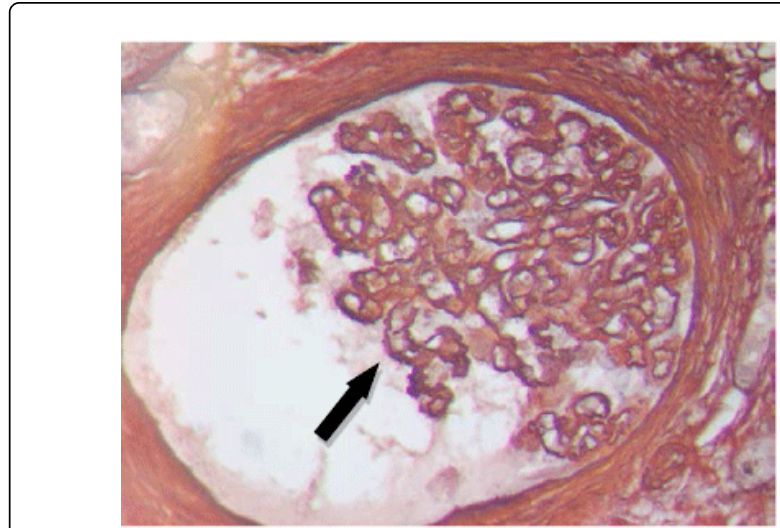

Figure 2: First kidney biopsy. Glomerulus demonstrating thickening of the basement membrane because of the basement membrane spike reaction around the deposits (Jones silver stain, $\times 400$ )

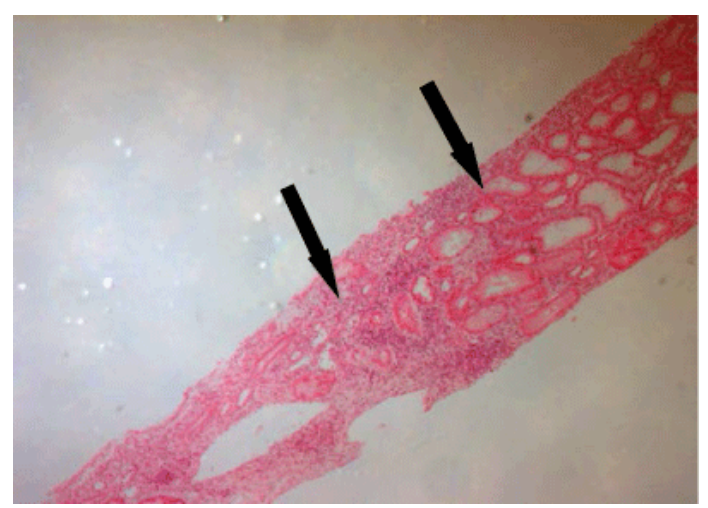

Figure 3: First kidney biopsy. Moderate tubular atrophy and interstitial infiltrates (periodic acid Schiff, $\times 40$ )

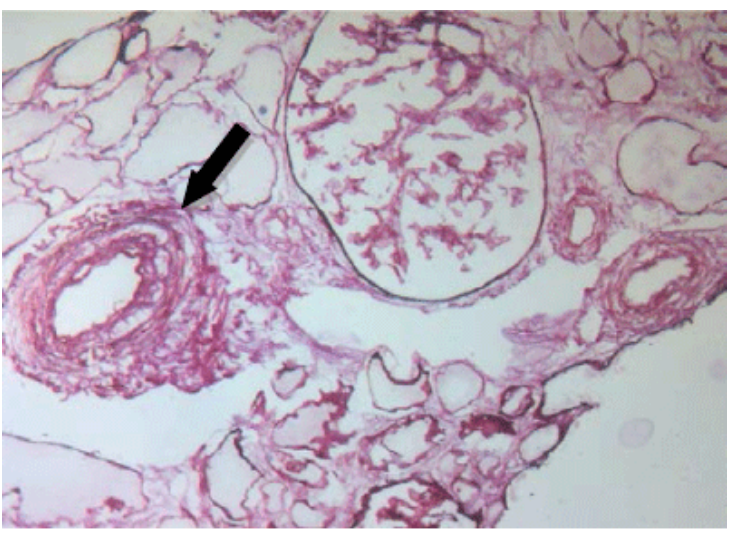

Figure 4: Second kidney biopsy. Arteriosclerosis, intimal fibroplasia of the interlobular artery (periodic acid Schiff, $\times 100$ ) 


\section{Discussion}

The antiphospholipid syndrome (APS) is an autoimmune disorder characterized by the occurrence of venous or arterial thrombosis or of specific pregnancy morbidity, in the presence of laboratory evidence of antiphospholipid antibodies (aPL). APS was first described by Hughes in the mid-1980s and it was in SLE that this syndrome was first reported [7]. But it is well known now that APS occurs either as a primary condition or in the setting of an underlying disease like SLE [8]. Our lupus patient had three criterias for APS: three miscarriages, the positive antiphospholipid antibodies and the deep venous thrombosis (DVT) that developed later in the course of her disease. And before the occurrence of the DVT, she was not treated for APS because she was allergic to aspirin and the kidney biopsy did not report any thrombotic lesions.

It is thus very important to recognize the entity called APSnephropathy (APSN). APSN refers to the kidney damage caused by vascular lesions in the glomeruli, arterioles and/or interlobular arteries in patients with aPL [9]. APSN vascular lesions may be acute, the socalled thrombotic microangiopathy (TMA), and/or chronic, such as arteriosclerosis, fibrous intimal hyperplasia, tubular thyroidization and focal cortical atrophy [10-12]. Although the two kidney biopsies in our patient did not mention the diagnosis of APSN, the vascular lesions of arteriosclerosis and the retracted appearance of one glomerulus tuft were very suggestive of a chronic vascular disease.

It is not rare to find aPL in lupus nephritis patients. APS seems also to be more prevalent in membranous lupus nephritis. In a review of 111 patients with LN followed for 15 years, the overall prevalence of antiphospholipid antibodies was 26 percent and it occurred more frequently in patients with membranous LN (50 versus 22 percent with other lesions) [10].

Although some authors would debate it [13], it is well accepted that morphologic lesions of APS-nephropathy may aggravate the lupus nephropathy $[10,14]$. APS occurs frequently in systemic lupus, and there is moderate-quality evidence that failure to treat it may lead to CKD or ESRD, despite adequate control of LN with immunosuppression [15]. APS was suspected to be the cause of the poor renal outcome of our patient because she did not receive at the beginning any treatment for APS and after she was put on oral anticoagulant she stabilized her renal function for several months. Previous reports have shown that anticoagulation treatment with international normalized ratio (INR) $>3$ may reverse renal artery stenosis as well and achieve subsequent clinical improvement [16]. And the latest KDIGO guidelines suggest to treat patients with LN and APS with anticoagulation in order to achieve an INR between 2-3 [15].

\section{Conclusion}

Nephrologists and nephropathologists should be more aware of the existence and the impact of APS nephropathy in LN patients. Anticoagulation introduced early is thought to be very effective in improving the renal outcome of those patients.

\section{References}

1. Weening JJ, D'Agati VD, Schwartz MM (2004) International Society of Nephrology Working Group on the Classification of Lupus Nephritis; Renal Pathology Society Working Group on the Classification of Lupus Nephritis. The classification of glomerulonephritis in systemic lupus erythematosus revisited. Kidney Int 65: 521-30.

2. [No authors listed] (1992) Lupus nephritis: prognostic factors and probability of maintaining life-supporting renal function 10 years after the diagnosis. Gruppo Italiano per lo Studio della Nefrite Lupica (GISNEL). Am J Kidney Dis 19: 473-479.

3. Sun HO, Hu WX, Xie HL, Zhang HT, Chen HP, et al. (2008) Long-term outcome of Chinese patients with membranous lupus nephropathy. Lupus 17: 56-61.

4. Mercadal L, Montcel ST, Nochy D, Queffeulou G, Piette JC, et al. (2002) Factors affecting outcome and prognosis in membranous lupus nephropathy. Nephrol Dial Transplant 17: 1771-1778.

5. Tektonidou MG, Sotsiou F, Nakopoulou L, Vlachoyiannopoulos PG, Moutsopoulos HM (2004) Antiphospholipid syndrome nephropathy in patients with systemic lupus erythematosus and antiphospholipid antibodies: prevalence, clinical associations, and long-term outcome. Arthritis Rheum 50: 2569-2579.

6. Moroni G, Ventura D, Riva P, Panzeri P, Quaglini S, et al. (2004) Antiphospholipid antibodies are associated with an increased risk for chronic renal insufficiency in patients with lupus nephritis. Am J Kidney Dis 43: $28-36$.

7. Alchi B, Griffiths M, Jayne D (2010) What nephrologists need to know about antiphospholipid syndrome. Nephrol Dial Transplant 25: 3147-3154.

8. Bobba RS, Johnson SR, Davis AM (2007) A review of the sapporo and revised Sapporo criteria for the classification of antiphospholipid syndrome. Where do the revised sapporo criteria add value? J Rheumatol 34: 1522-1527.

9. Dekeyser M, Zuily S2, Champigneulle J3, Eschwège V4, Frimat L5, et al. (2014) [Antiphospholipid syndrome in nephrology. Kidney damage and practical aspects of the management]. Nephrol Ther 10: 1-9.

10. Daugas E, Nochy D, Huong DL, Duhaut P, Beaufils H, et al. (2002) Antiphospholipid syndrome nephropathy in systemic lupus erythematosus. J Am Soc Nephrol 13: 42-52.

11. Tektonidou MG (2009) Renal involvement in the antiphospholipid syndrome (APS)-APS nephropathy. Clin Rev Allergy Immunol 36: 131-140.

12. Nochy D, Daugas E, Droz D, Beaufils H, Grünfeld JP, et al. (1999) The intrarenal vascular lesions associated with primary antiphospholipid syndrome. J Am Soc Nephrol 10: 507-518.

13. Erre GL, Bosincu L, Faedda R, Fenu P, Masala A, et al. (2014) Antiphospholipid syndrome nephropathy (APSN) in patients with lupus nephritis: a retrospective clinical and renal pathology study. Rheumatol Int 34: 535-541.

14. Asherson RA, Klumb EM (2008) Antiphospholipid syndrome nephropathy in different scenarios. J Rheumatol 35: 1909-1911.

15. Kidney Disease: Improving Global Outcomes (KDIGO) 2012 Glomerulonephritis Work Group. KDIGO Clinical Practice Guideline for Glomerulonephritis. Kidney inter., Suppl. 2: 139-274.

16. Sangle SR, D'Cruz DP, Abbs IC (2005) Renal artery stenosis in hypertensive patients with antiphospholipid (Hughes) syndrome: outcome following anticoagulation. Rheumatology (Oxford) 44: 372-377. 